\title{
AiMT
}

Advances in Military Technology

Vol. 15, No. 1, 2020, pp. 163-178

ISSN 1802-2308, eISSN 2533-4123

DOI 10.3849/aimt.01314

\section{Automatic Control of an Asymmetric Fighter Aircraft Performing Supermanoeuvres}

\author{
B.K. Mukherjee ${ }^{1 *}$, K. Goel ${ }^{2}$ and M. Sinha ${ }^{2}$ \\ ${ }^{1}$ Department of Electrical and Electronics Engineering, BITS Pilani, India \\ ${ }^{2}$ Department of Aerospace Engineering, IIT Kharagpur, India
}

The manuscript was received on 5 March 2019 and was accepted after revision for publication as research paper on 28 March 2020.

\begin{abstract}
:
Centre-of-gravity (c.g.) of combat aircraft suffers significant lateral deviation due to asymmetric release of stores, leading to a highly nonlinear and coupled dynamics. Additional nonlinearity and coupling result when the aircraft attempts some supermanoeuvres under such conditions rendering nonlinear control implementation unavoidable. However, such controls depend on accurate onboard c.g information. The present paper proposes a novel neural network aided sliding mode based hybrid control scheme which does not require such an information. The neural controller is trained offline to compensate for the changed dynamics arising from the lateral mass asymmetry, while the sliding controller is designed for the intended manoeuvres under the nominal situation. Cobra and Herbst manoeuvres are simulated for various lateral c.g. movements to validate the scheme.
\end{abstract}

\section{Keywords:}

centre-of-gravity, neural network, sliding mode, supermanoeuvre

\section{Introduction}

Aircraft having lateral asymmetry in inertial and aerodynamic properties inherent in their structure have recently gained prominence in aircraft flight dynamics and control research [1-9]. In civil aircraft, such an asymmetry may generally arise due to asymmetry in fuel consumption, partial structural damage in the wing etc. Initial works in this direction focused on civil aircraft only trying to maintain steady level flight under partial wing damage. However, significantly asymmetric dynamics may also occur in fighter aircraft because of asymmetric firing of stores. Leaving the dynamics asymmetric has the advantage of not having to carry dummies or stores in identical pairs. Moreover, such an asymmetric fighter aircraft may be forced to execute some complex

\footnotetext{
* Corresponding author: Department of Electrical and Electronics Engineering, BITS Pilani, Rajasthan IN-333031, India. E-mail: bjoy.here@gmail.com
} 
and rapid manoeuvres because of mission and/or safety demands. Thus, the aircraft must be equipped so that they can complete the mission and/or safely return to the base even when these asymmetries have occurred. In many cases the asymmetry induced due to the aforementioned reasons cannot be accurately identified and quantified. For example, identification of the asymmetry due to firing of stores needs online centre of gravity estimation in order for the controllers to be able to compensate for the changed dynamics, as considered recently in the literature [5-9]. Further, the changed dynamics is far more complex and control design based on them requires considerably more online computation power. The present paper attempts to remove such restrictions on online c.g. information, as well as increased computation power requirement altogether so that a fixed parameter control scheme can effectively handle a wide range of asymmetric c.g. variations resulting from arbitrarily asymmetric release of stores.

During the standard fighter aircraft manoeuvres, the aircraft is usually required to pitch up to a very high angle forcing it to operate in the high angle of attack regions, i.e. regions far exceeding the stall limit. In such regions, the aircraft flight dynamics becomes predominantly nonlinear due to aerodynamic, inertial and trigonometric effects [10]. The situation further aggravates when additional nonlinearity and coupling between longitudinal and lateral-directional dynamics surface because of the lateral mass asymmetry. As a result, implementation of nonlinear control techniques becomes indispensable. Two well-known nonlinear controller design techniques, namely Nonlinear Dynamic Inversion (NDI) and Sliding Mode Control (SMC) have been conventionally applied to various aircraft flight control problems in the past [10-12]. In the present work, however, SMC is preferred because of its inherent robustness properties to structured uncertainties.

In the proposed scheme, a sliding controller (denoted henceforth as the Nominal SMC) is designed for the symmetric aircraft (or the nominal aircraft) using the standard symmetric six degree of freedom (6-DOF) dynamics for the faster inner loop involving angular rates. This inner loop controller is aided by a Multilayer Feedforward Neural Network (MFNN) controller (as shown in Fig. 1) to take care of the changes in the dynamics arising from the lateral c.g. shift. The MFNN controller is trained offline considering a few c.g. movement values within a feasible range. The asymmetric aircraft equation of motion as derived in the literature $[1,2,7]$ is exploited in generating the training data for the offline training of the neural network. Neural networks have been previously used in similar dynamic situations [2, 13, 14], however the proposed formulations therein contain an online neural network with update of weights being done online. The present controller, on the other hand, is a fixed parameter one and therefore does not demand additional online computation power. The slower outer loop containing the angular variables is predominantly kinematic in nature and therefore it does not get directly affected by the changes in the dynamics from the mass asymmetry. Hence, the outer loop controller is only a nominal SMC controller designed considering the nominal (i.e. symmetric) outer loop dynamics.

Section 2 gives the details of the proposed hybrid control scheme. To validate the usefulness of the proposed controller formulation, two standard supermanoeuvres cobra and Herbst are considered in the present paper. In cobra, the aircraft suddenly pitches up to about ninety degrees or sometimes even more and then immediately comes down to the initial pitch within a few seconds. As a result, the aircraft suddenly brakes to a much lower velocity forcing the pursuing aircraft to overshoot [15]. In Herbst manoeuvre, the aircraft first pitches rapidly to a high angle of attack and there- 
after initiates banking at a high rate to quickly reverse the flight direction $[16,17]$. In the present scenario of significant lateral asymmetry, Herbst manoeuvre is particularly relevant since the aircraft can at least abort the mission and safely return to the base. Simulation results showing the manoeuvre performance for these manoeuvres over a considerable lateral c.g. offset range are presented in Section 3. Section 4 concludes the paper.

\section{Neural Network Aided Hybrid Sliding Mode Control Scheme}

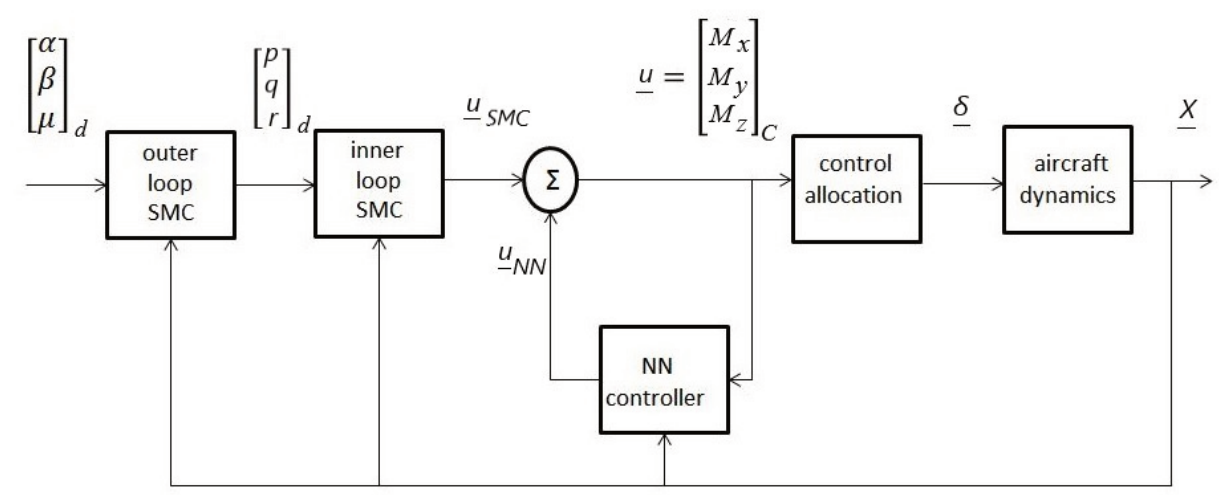

Fig. 1 Block diagram of the proposed closed loop control scheme

Sliding Mode Control is a robust nonlinear controller design technique particularly suitable for dealing with structured or parametric uncertainties [18-19]. This control approach is found to mitigate a wide range of uncertainties in the case of asymmetric aircraft [7-9]. However, the manoeuvre performance considerably deteriorates when the controller does not have an accurate information of the asymmetry. Since the angular and angular rate dynamics are timescale separated, usually two separate controllers are designed for them in an inner-outer loop architecture, as shown above in Fig. 1. The same architecture is retained in the present scheme with an additional aid coming from the neural controller in the inner loop. The 6-DOF equations of motion for asymmetric aircraft as given in the Appendix can be simplified as

$$
\begin{array}{r}
{\left[\begin{array}{c}
\dot{p} \\
\dot{q} \\
\dot{r}
\end{array}\right]=\left(\frac{1}{m^{\prime}}[\mathbf{A}]^{2}+\boldsymbol{I}^{\prime}\right)^{-1}\left(-[\mathbf{A}]\left[\begin{array}{l}
F_{1} \\
F_{2} \\
F_{3}
\end{array}\right]-\frac{1}{m^{\prime}}[\mathbf{A}]\left[\begin{array}{l}
F_{x} \\
F_{y} \\
F_{z}
\end{array}\right]+\left[\begin{array}{l}
M_{1} \\
M_{2} \\
M_{3}
\end{array}\right]+\left[\begin{array}{l}
M_{x} \\
M_{y} \\
M_{z}
\end{array}\right]_{\mathrm{S}}+\left[\begin{array}{l}
M_{x} \\
M_{y} \\
M_{z}
\end{array}\right]_{\mathrm{C}}\right)} \\
{\left[\begin{array}{c}
\dot{u} \\
\dot{v} \\
\dot{w}
\end{array}\right]=\frac{1}{m^{\prime}}[\mathbf{A}]\left(\frac{1}{m^{\prime}}[\mathbf{A}]^{2}+\boldsymbol{I}^{\prime}\right)^{-1}\left(-[\mathbf{A}]\left[\begin{array}{l}
F_{1} \\
F_{2} \\
F_{3}
\end{array}\right]-\right.} \\
\left.-\frac{1}{m^{\prime}}[\mathbf{A}]\left[\begin{array}{l}
F_{x} \\
F_{y} \\
F_{z}
\end{array}\right]+\left[\begin{array}{l}
M_{1} \\
M_{2} \\
M_{3}
\end{array}\right]+\left[\begin{array}{l}
M_{x} \\
M_{y} \\
M_{z}
\end{array}\right]_{\mathrm{S}}+\left[\begin{array}{l}
M_{x} \\
M_{y} \\
M_{z}
\end{array}\right]_{\mathrm{C}}\right)+\left[\begin{array}{l}
F_{1} \\
F_{2} \\
F_{3}
\end{array}\right]+\frac{1}{m^{\prime}}\left[\begin{array}{l}
F_{x} \\
F_{y} \\
F_{z}
\end{array}\right]
\end{array}
$$

where $\left[M_{x} M_{y} M_{z}\right]_{\mathrm{s}}^{\mathrm{T}}$ and $\left[M_{x} M_{y} M_{z}\right]_{\mathrm{c}}^{\mathrm{T}}$ denote the state and control dependent components of the external moment vector, and 


$$
[\mathbf{A}] \triangleq\left[\begin{array}{ccc}
0 & -m^{\prime} z_{\mathrm{cm}} & m^{\prime} y_{\mathrm{cm}} \\
m^{\prime} z_{\mathrm{cm}} & 0 & -m^{\prime} x_{\mathrm{cm}} \\
-m^{\prime} y_{\mathrm{cm}} & m^{\prime} x_{\mathrm{cm}} & 0
\end{array}\right]
$$

and $\left[\begin{array}{lll}F_{1} & F_{2} & F_{3}\end{array}\right]^{\mathrm{T}}$ denotes the first and third terms combined on the right hand side of the force balance equation and $\left[\begin{array}{lll}M_{1} & M_{2} & M_{3}\end{array}\right]^{\mathrm{T}}$ denotes the first three terms combined on the right hand side of the moment balance equation, as given in the Appendix. $m^{\prime}$ and $\boldsymbol{I}^{\prime}$ are the post ejection mass and moment of inertia of the aircraft and $\left[\begin{array}{ll}x_{\mathrm{cm}} & y_{\mathrm{cm}} z_{\mathrm{cm}}\end{array}\right]^{\mathrm{T}} \mathrm{de}-$ notes the location of the c.g. from the centre of the body frame.

On the other hand, nominal dynamics for an aircraft is given by the usual 6-DOF equations of motion again given in the Appendix for reference. Now let the asymmetric rotational dynamics in Eq. (1) be described as

$$
\begin{gathered}
\dot{x}_{1}=f(x)+g(x) u \\
\dot{x}_{1}=f_{0}(x)+g_{0}(x) u+\{\Delta f(x)+\Delta g(x) u\} \\
\dot{x}_{1}=f_{0}(x)+g_{0}(x) u+\Delta F(x, u)
\end{gathered}
$$

where the part $\left\{f_{0}(x)+g_{0}(x) u\right\}$ represents the nominal or symmetric angular rate dynamics, control signal $u=\left[M_{x} M_{y} M_{z}\right]_{\mathrm{c}}^{\mathrm{T}}, x_{l}=\left[\begin{array}{ll}p & q\end{array}\right]^{\mathrm{T}}$ and $x$ is the state vector. Clearly,

$$
\Delta F(x, u)=\{f(x)+g(x) u\}-\left\{f_{0}(x)+g_{0}(x) u\right\}
$$

The aim of the neural network controller is to learn the difference in the nominal dynamics and the asymmetric dynamics, i.e. the $\Delta F(x, u)$ term for different levels of mass asymmetry in an offline manner. In order to make the controller independent of the store weight that is being fired or the amount of c.g. variation, a Multilayer FeedForward Neural Network (MFNN) based controller is proposed. This approach is, indeed, appealing because of the excellent capabilities of the feedforward neural networks for adapting to various changes in its input parameters even if the dimensionality of the data to be learnt is too high. Following the formulations as proposed in [8, 9], first an SMC controller is designed using the actual asymmetric dynamics. From this simulation, the $\Delta F(x, u)$ values are stored as per Eq. (7). These values are used for offline training of the MFNN controller.

Let a fully trained three layer network (one input layer, one hidden layer and one output layer) with optimal weight matrices $\boldsymbol{W}$ and $\boldsymbol{V}$ represent the function $\Delta F(x, u)$ with some approximation error $\varepsilon$ bounded as $-\varepsilon_{\mathrm{m}} \leq \varepsilon \leq \varepsilon_{\mathrm{m}}$. Therefore,

$$
\Delta F(x, u)=\boldsymbol{W}^{\mathrm{T}} \phi(\boldsymbol{V}, x, u)+\varepsilon
$$

where $\phi$ is the activation function of the hidden layer and $\boldsymbol{V}$ is the weight matrix of the hidden layer; and $\boldsymbol{W}$ is the weight matrix of the output layer. Now, substituting Eq. (8) in Eq. (6), the asymmetric rate dynamics can be described as

$$
\dot{x}_{1}=f_{0}(x)+g_{0}(x) u+\boldsymbol{W}^{\mathrm{T}} \boldsymbol{\phi}(\boldsymbol{V}, x, u)+\varepsilon
$$

As shown in Fig.1, the outer loop controller treats the body rates as the virtual control signals. The outer loop happens to be predominantly kinematic, therefore the mass asymmetry does not affect it much; it affects mostly the inner loop performance. Therefore, the neural controller is used to aid the inner loop SMC only so that the 
$\Delta F(x, u)$ part is compensated. Thus, the final control input to the plant representing the three moments about the three body axes is

$$
u=u_{\mathrm{SMC}}+u_{\mathrm{NN}}
$$

It is to be noted that the SMC part in Eq. (10) comes from the nominal SMC as designed for the symmetric dynamics and it proceeds in the standard manner. For faster convergence to the sliding surface, a linear term is added to a discontinuous term in the reaching law. Hence the reaching law is given by

$$
\dot{s}=-\eta_{1} \operatorname{sgn}(s)-\eta_{2} s
$$

where $\eta_{1}$ and $\eta_{2}$ are positive constants and the sliding surface is $s=0$ where $s$ containing proportional and integral components is chosen to be

$$
s=\left(x_{1 d}-x_{1}\right)+k_{1} \int\left(x_{1 d}-x_{1}\right) \mathrm{d} t
$$

where $x_{1 \mathrm{~d}}$ denotes the desired profile of $x_{1}$ and $k_{1}$ is a positive constant. The sliding controller output is computed as

$$
u_{\mathrm{SMC}}=\frac{1}{g_{0}(x)}\left[-f_{0}(x)-k_{1} x_{1}+\left(\dot{x}_{1 \mathrm{~d}}+k_{1} x_{1 \mathrm{~d}}\right)\right]+\eta_{1} \operatorname{sgn}(s)+\eta_{2} s
$$

If the asymmetric dynamics were known to the SMC controller at each time step, then the control moment $u$ would have been computed from

$$
u_{\mathrm{SMC}}=\frac{1}{g_{0}(x)}\left[-f_{0}(x)-\Delta F-k_{1} x_{1}+\left(\dot{x}_{1 \mathrm{~d}}+k_{1} x_{1 \mathrm{~d}}\right)\right]+\eta_{1} \operatorname{sgn}(s)+\eta_{2} s
$$

An estimate of the $\Delta F$ term, hereafter denoted as $\hat{\Delta} F$, is obtained from the neural controller. Therefore, the neural controller output is given by

$$
u_{\mathrm{NN}}=-\frac{1}{g_{0}(x)}[\hat{\Delta} F(x, u)]
$$

The estimation error in the MFNN controller $\varepsilon$ is small and is handled by the nominal SMC controller. Therefore, the dynamics as seen by the nominal SMC is given by

$$
\dot{x}_{1}=f_{0}(x)+g_{0}(x) u_{\mathrm{SMC}}+\mathcal{E}
$$

For the chosen sliding surface as given in Eq. (12),

$$
s \dot{s}=s\left(\dot{x}_{1 \mathrm{~d}}-\dot{x}_{1}+k_{1} x_{1 \mathrm{~d}}-k_{1} x_{1}\right)
$$

Substituting $\dot{x}$ from Eq. (16) and $u$ smC from Eq. (13) into Eq. (17) yields

$$
s \dot{s}=-s\left(\varepsilon+\eta_{1} \operatorname{sgn}(s)+\eta_{2} s\right)
$$

For stability of the sliding surface and for a minimum guaranteed convergence rate of the trajectory to the sliding surface, it is required that the following inequality is satisfied

$$
s \dot{s} \leq-k|s|
$$

where $k$ is a positive constant. Eqs (18) and (19) yield the condition 


$$
\begin{gathered}
-s\left(\varepsilon+\eta_{1} \operatorname{sgn}(s)+\eta_{2} s\right) \leq-k|s| \\
\eta_{1}|s|+\eta_{2} s^{2} \geq-\varepsilon s+k|s|
\end{gathered}
$$

From inequality (21) it is clear that for negative values of $s$, the worst scenario occurs when $\varepsilon=+\varepsilon_{\mathrm{m}}$ and for positive $s$, the worst situation arises when $\varepsilon=-\varepsilon_{\mathrm{m}}, \varepsilon_{\mathrm{m}}$ being the norm bound on the approximation error. Clearly, if the constant $\eta_{1}$ is chosen to satisfy the condition

$$
\eta_{1} \geq \varepsilon_{\mathrm{m}}+k
$$

then the inequality in (21) is always satisfied for all values of $s$ and for any positive value of the parameter $\eta_{2}$.

To reduce chattering in SMC, the hard signum function is usually replaced with a saturation function having a small linear region, say $\pm b$. Hence, Eq. (11) reduces to

$$
\dot{s}=-\eta_{1} \operatorname{sat}(s)-\eta_{2} s
$$

Substituting $s$ from Eq. (12) in Eq. (23),

$$
\dot{e}+k_{1} e=-\eta_{1} \operatorname{sat}(s)-\eta_{2} s
$$

where $e$ represents the tracking error in the state variable $x$ i.e. $x_{1 \mathrm{~d}}-x_{1}$. Therefore, in the linear region of the saturation function,

$$
\dot{e}+k_{1} e=-\eta_{1} \frac{1}{b} s-\eta_{2} s
$$

Substituting s from Eq. (12) in the right hand side of Eq. (25),

$$
\dot{e}+k_{1} e=-\left(\frac{\eta_{1}}{b}+\eta_{2}\right)\left(e+k_{1} \int e \mathrm{~d} t\right)
$$

Differentiating both sides of Eq. (26) once w.r.t. time yields

$$
\begin{gathered}
\ddot{e}+k_{1} \dot{e}+\left(\frac{\eta_{1}}{b}+\eta_{2}\right) \dot{e}+\left(\frac{\eta_{1}}{b}+\eta_{2}\right) k_{1} e=0 \\
\ddot{e}+\left(\frac{\eta_{1}}{b}+\eta_{2}+k_{1}\right) \dot{e}+\left(\frac{\eta_{1}}{b}+h_{2}\right) k_{1} e=0
\end{gathered}
$$

The control parameters are chosen in such a manner that the above error dynamics is sufficiently faster than the corresponding error dynamics for the outer loop. This is done to ensure that the inherent time scale separation between the inner and outer loops is retained.

In the present scheme, suitable desired profiles for the angle-of-attack, sideslip angle and the bank angle i.e. $\alpha, \beta$ and $\mu$ are fed to the outer loop controller (as shown in Fig. 1) for executing the intended manoeuvres. The outer loop sliding mode controller, which generates the desired body rates i.e. $x_{1 \mathrm{~d}}$, is designed in the conventional way neglecting the asymmetric dynamics altogether [7, 8]. Further, the control moments demanded from the controller are converted to control surface deflection commands through the standard pseudo-inverse control allocation method [10] and availability of full state feedback is assumed. 


\section{Simulation Results and Discussion}

To validate the proposed hybrid controller, in the present work, cobra and Herbst manoeuvres are simulated considering various amounts of lateral c.g. variations. The amount of lateral c.g. variation is represented in terms of the mass of the store, which is supposed to be located at a fixed location of one third of the semispan from the fuselage centre line. Mass of the store is considered to be within the range from zero to $1100 \mathrm{~kg}$ at an interval of $100 \mathrm{~kg}$. This represents a feasible range of lateral c.g. variations (of about 0 to $9 \mathrm{~cm}$ ). This introduces a significant asymmetry in the 6-DOF dynamics of the aircraft and as reported in the recent literature $[7,8]$, the controller should be designed on the asymmetric dynamics if the desired manoeuvre performance is to be retained. This required the controller to know the actual c.g. location. However, in the present scheme, neither of the neural and sliding controllers is fed any information about this c.g. shift. The manoeuvre durations considered are $6 \mathrm{~s}$ and $18 \mathrm{~s}$ for cobra and Herbst respectively.

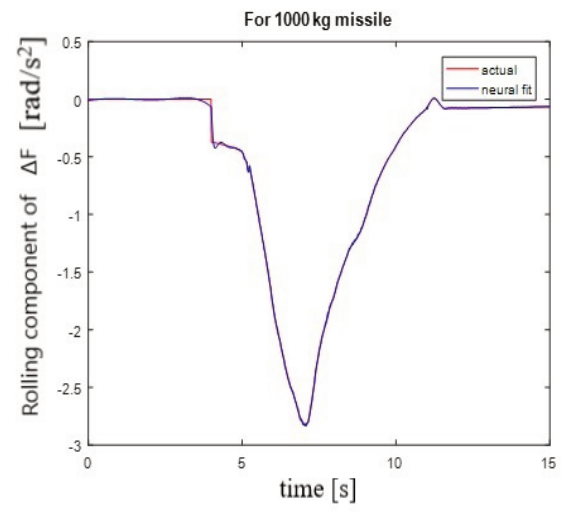

(a)

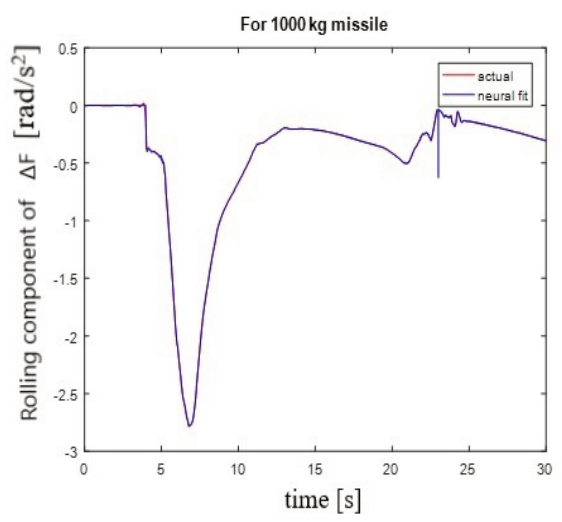

(b)

Fig. 2 Rolling accelerations induced due to asymmetry from firing a $1000 \mathrm{~kg}$ store: (a) Cobra (b) Herbst

To generate the training data set for offline training of the MFNN controller, first the SMC is designed using the asymmetric dynamic equations (as detailed in [7]) for three cases of store mass: $200 \mathrm{~kg}, 600 \mathrm{~kg}$ and $1000 \mathrm{~kg}$. In each case, from the simulations, the $\Delta F(x, u)$ values are computed and saved (refer to Eq. (7)). Since the neural controller is designed for the inner loop, $\Delta F(x, u)$ basically has three angular acceleration components. All the three data sets corresponding to three different store masses are used to train the offline neural network controller. One hidden layer is considered with 15 and 25 neurons for cobra and Herbst manoeuvre cases respectively.

Training takes about 412 and 623 iterations respectively and the maximum tolerance for the mean square error is set $10^{-5}$. Levenberg-Marquardt algorithm is used for training the network using the MATLAB ${ }^{\mathrm{TM}}$ Neural Networks Toolbox [21]. Fig. 2 shows fits for the rolling acceleration component induced when a $1000 \mathrm{~kg}$ store is released. The $\Delta F(x, u)$ term contains pitch and yaw components also, however, their neural network fits are not shown for the sake of brevity. Moreover, the uncertainty is 
predominantly in the rolling component. It is assumed that the store is released at $t=4 \mathrm{~s}$ and the manoeuvre is initiated at $t=5 \mathrm{~s}$.

The hybrid controller is validated for all twelve cases, i.e. for store mass ranging from zero to $1100 \mathrm{~kg}$ at an interval of $100 \mathrm{~kg}$. Fig. 3 shows simulation results for an intermediate case (i.e. a situation which is not considered for training the neural network controller) when the aircraft executes the cobra manoeuvre after a $900 \mathrm{~kg}$ store is released. Time response for three controllers are compared: i) the Nominal SMC designed neglecting the asymmetric dynamics completely; ii) Exact SMC designed using the asymmetric dynamics and iii) the proposed MFNN-SMC hybrid controller. The aircraft is commanded to pitch up to $90^{\circ}$ within $3 \mathrm{~s}$ and then to come back to initial pitch in other $3 \mathrm{~s}$.

From Fig. 3 it can be readily observed that the performance of the MFNN-SMC controller is very close to the Exact SMC controller, while the Nominal SMC controller performance is significantly inferior - the aircraft suffering wide lateral deviation in its trajectory and pitch angle going up to less than $80^{\circ}$ instead of the commanded $90^{\circ}$. The poor performance of the Nominal SMC controller implies that the asymmetry in the dynamics cannot be completely neglected (even though a robust controller like the sliding mode is used) while attempting such high performance manoeuvres automatically. However, if the exact asymmetric equations are used to design the SMC controller, then c.g position should be fed to the controller. On the other hand, the proposed hybrid controller achieves almost identical performance level with no such need for the c.g. information. Moreover, the MFNN-SMC scheme is a fixed parameter control scheme as both the MFNN and SMC parameters are set offline. Simulations are tested for any arbitrary store mass in the range of zero to $1100 \mathrm{~kg}$ and the same manoeuvre performance is found to be retained. Results for only one case are presented here in Fig. 3.

Fig. 4 shows the performance of the three aforementioned controllers for Herbst manoeuvre. The first plot in Fig. 4 shows the trajectory of the aircraft; from this figure, it is clear that the Nominal SMC controller completely fails to execute the manoeuvre as the aircraft flies west instead of south after the manoeuvre. The Exact SMC controller and the proposed MFNN-SMC controller performances are nearly identical - the aircraft reversing its flight direction nearly perfectly in either case. However, the computation time for the Exact SMC control scheme is about $30 \%$ higher than that of the proposed MFNN-SMC control scheme. The aircraft is assumed to possess conventional aerodynamic control surfaces and thrust vector controls in both pitch and yaw planes. All the aerodynamic and thrust control surfaces are limited by position and rate limits as given in the Appendix. For simulating the manoeuvres, the aircraft's aerodynamic coefficients are considered to have a random uncertainty of $\pm 10 \%$ about their nominal values in case of Herbst manoeuvre and $\pm 25 \%$ in case of cobra manoeuvre. Since the Herbst manoeuvre is much more demanding, a higher uncertainty level could not be handled by the sliding mode controller. To reduce chattering, a saturation function is used with a small linear region $( \pm 0.05)$ instead of a signum function. The $\eta_{1}$ and $\eta_{2}$ values are set at 0.1 and 0.01 respectively.

The commanded and actual AOA profiles while using the proposed hybrid MFNN-SMC controller are shown in Fig. 5a for cobra manoeuvre. For Herbst manoeuvre, both AOA and bank angles are of importance; hence both profiles are shown in Fig. 5b. Fig. 5 reveals that the proposed controller achieves excellent tracking of the commanded profiles in both the cases. The three-dimensional trajectories of the aircraft for the two manoeuvres are shown in Fig. 6. 

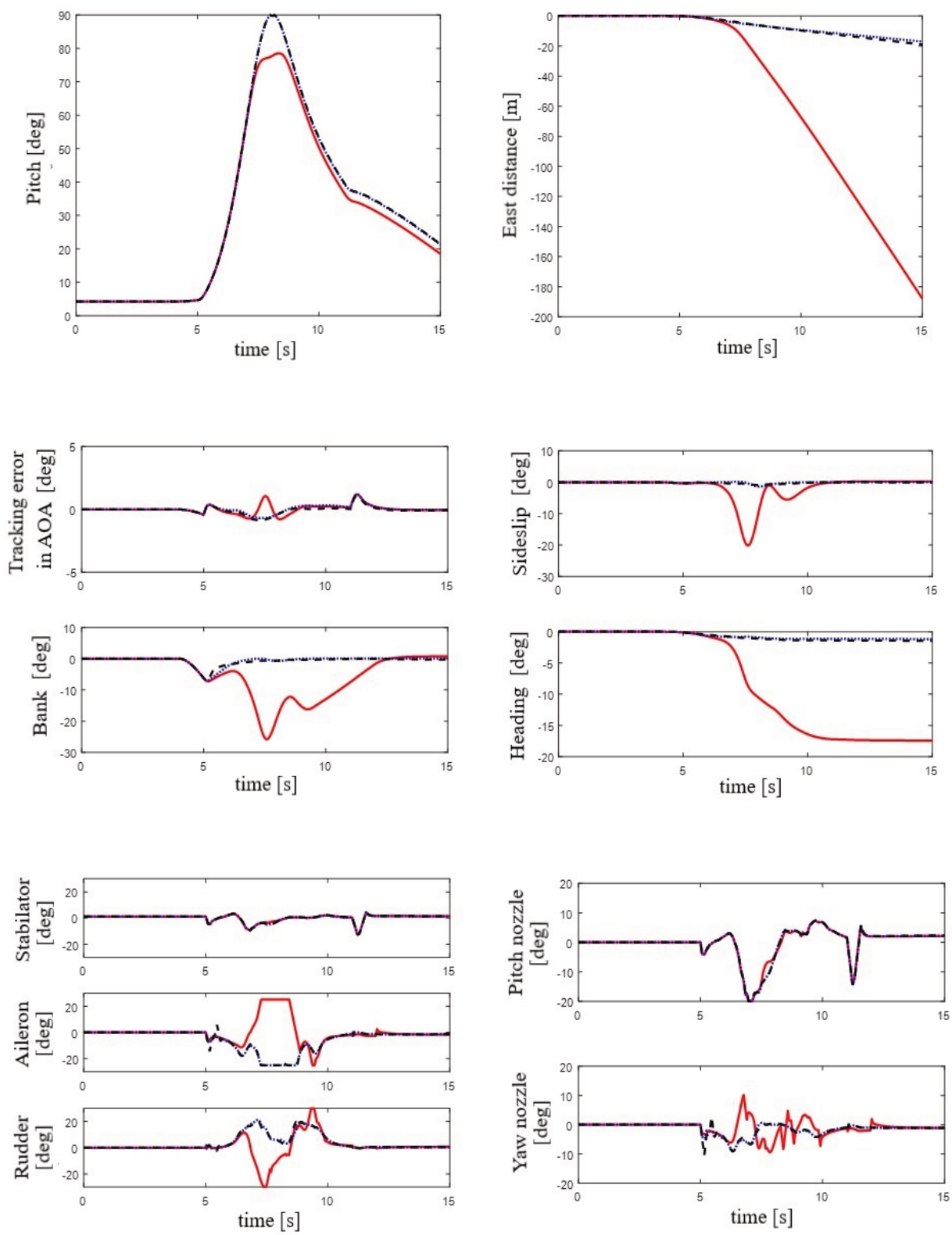

Fig. 3 Cobra manoeuvre response (Nominal SMC - Red solid, Exact SMC - Blue dotted and MFNN-SMC Hybrid Controller - Black dashed) 

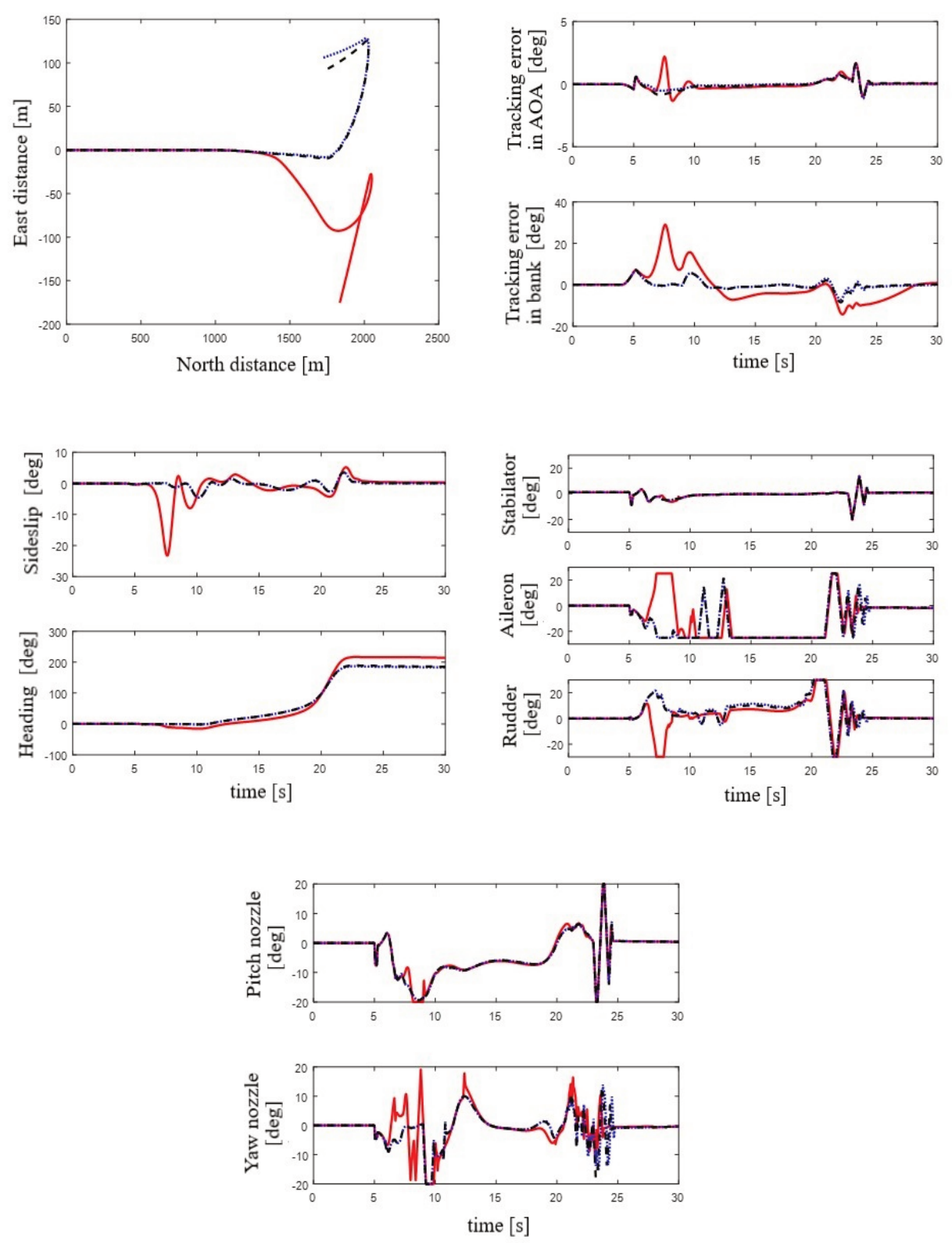

Fig. 4 Herbst Manoeuvre response (Nominal SMC - Red solid,

Exact SMC - Blue dotted and MFNN-SMC Hybrid Controller - Black dashed) 


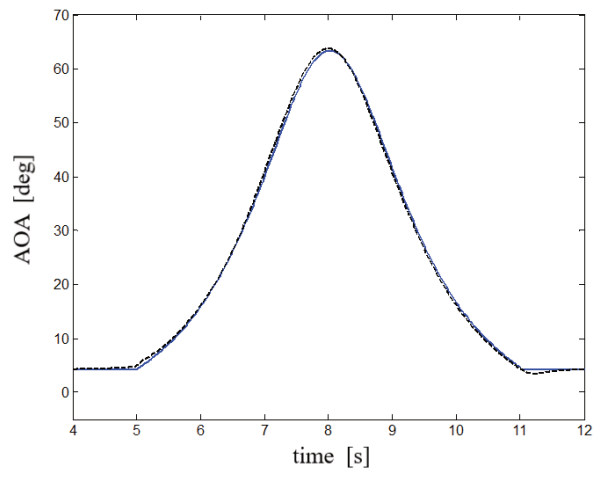

(a)

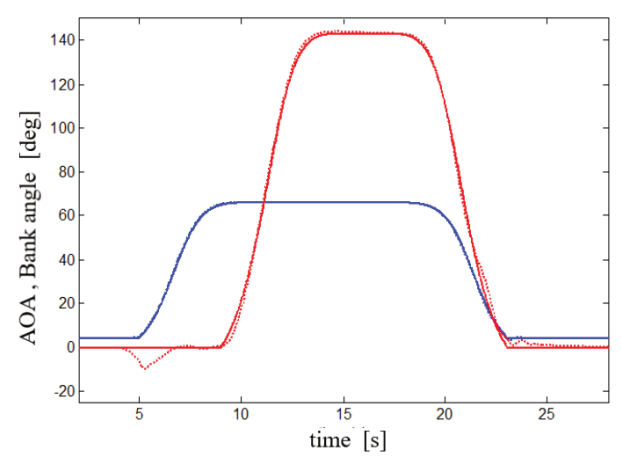

(b)

Fig. 5 (a) AOA profile for Cobra Manoeuvre (Commanded-solid, Actual using the MFNN-SMC Hybrid Controller - dotted); (b) AOA (in blue) and bank angle (in red) profiles for Herbst Manoeuvre (Commanded - solid, Actual using the MFNN-SMC Hybrid Controller - dotted)

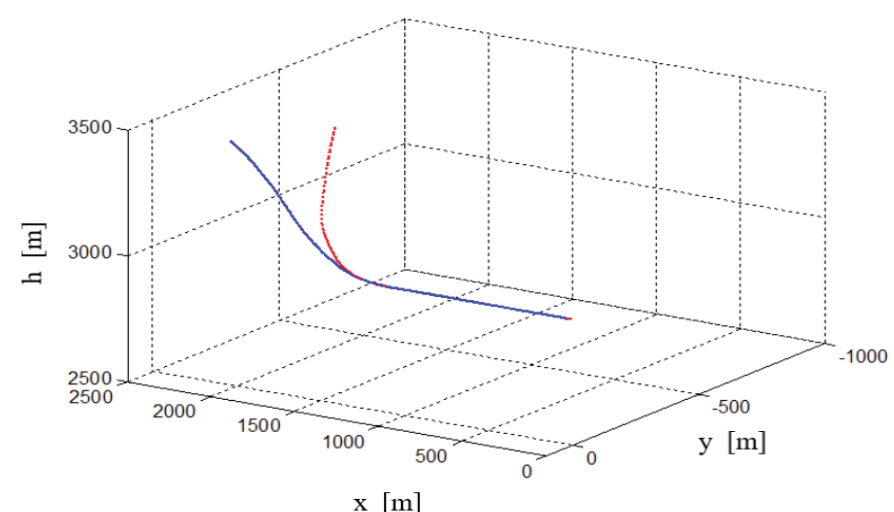

(a)

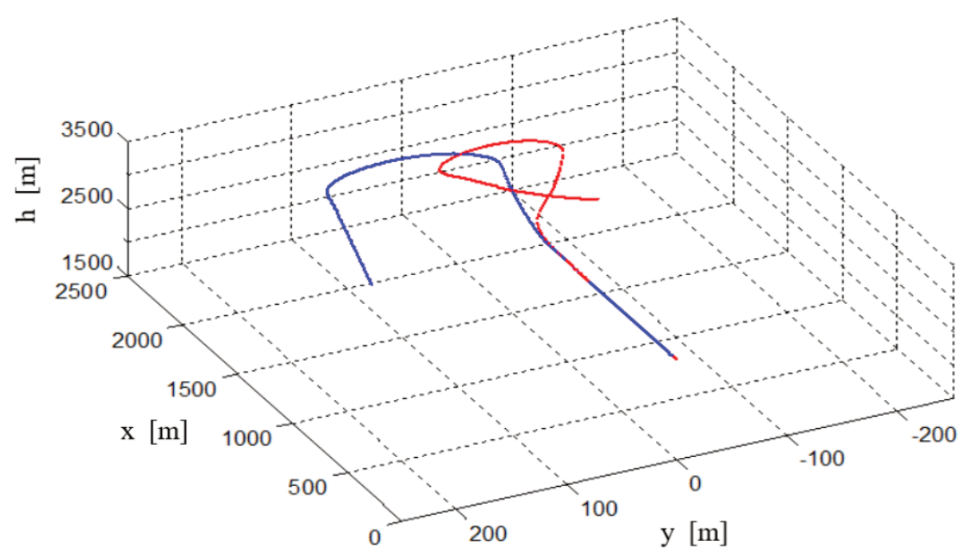

(b)

Fig. 6 3-D Trajectory (a) Cobra Manoeuvre, (b) Herbst Manoeuvre (Nominal SMC-Red and MFNN-SMC Hybrid Controller - Blue) 
For the cobra manoeuvre case, it is observed that the proposed controller does not result in any noticeable lateral deviation of the aircraft (blue plot in Fig. 6a) whereas if the Nominal SMC were used, the aircraft would have undergone significant lateral deviation (red plot in Fig. 6a). For Herbst manoeuvre, the proposed MFNN-SMC controller reverses the flight direction by $180^{\circ}$ as the aircraft flies along the negative $\mathrm{x}$-direction after the manoeuvre is executed (blue plot in Fig. 6b). However, if the Nominal SMC were retained, the aircraft would fly along the negative y-direction instead of the desired negative x-direction after the manoeuvre is completed (red plot in Fig. 6b). In Fig. 6, positive x-direction represents the forward direction of flight.

\section{Conclusion}

The paper presented a novel hybrid control architecture combining feedforward neural network and sliding mode techniques for performing supermanoeuvres with an aircraft under significant lateral mass asymmetry assuming no estimation of the asymmetry on board. Results showed that performance of the proposed scheme provided significantly improved results in comparison with a standard sliding control formulation which ignores the changes in aircraft dynamics arising from the mass asymmetry. It was also observed that if the sliding controller was designed considering the asymmetric dynamics explicitly, then manoeuvre performance of the same level as that of the proposed control scheme would be achieved but that would require online c.g. information along with significantly more complexity in control computations. Future work in this direction may entail inclusion of asymmetry due to partial structural damage in the wing along with the asymmetry in the lateral mass distribution.

\section{APPENDIX}

\section{Notations and Abbreviations}

$V, h$
$\alpha, \beta$
$\varphi, \theta, \psi$
$\mu, \gamma, \chi$
$p, q, r$
$u, v, w$
$F_{x}, F_{y}, F_{z}$
$M_{x}, M_{y}, M_{z}$
$m, m^{\prime}$
$I_{x x}, I_{y y}, I_{z z}, I_{x z}$
$I_{x x}^{\prime}, I_{y y}^{\prime}, I_{z z}^{\prime}, I_{x y}^{\prime}, I_{y z}^{\prime} I_{x z}^{\prime}$
$g$
$x_{\mathrm{cm}}, y_{\mathrm{cm}}, z_{\mathrm{cm}}$
$\delta_{e}, \delta_{a}, \delta_{r}$
$\delta_{T}, \delta_{p}, \delta_{y}$
$x_{1}$
$x_{1 \mathrm{~d}}$
$e$

Velocity, altitude angle-of-attack (AOA) and sideslip angle roll, pitch and yaw Euler angles bank, flight path and heading angles body-axis roll, pitch and yaw rates velocity components along body $\mathrm{x}-\mathrm{y}-\mathrm{z}$ axes external forces along the three body axes external moments along the three body axes mass of the aircraft pre and post ejection of the store moments and product of inertia before ejection of the store moments and products of inertia after ejection of the store acceleration due to gravity c.g. location from the origin of the body frame stabilator, aileron and rudder deflections throttle, pitch and yaw nozzle deflections $\left[\begin{array}{lll}p & q & r\end{array}\right]^{\mathrm{T}}$ desired value of $x_{1}$ tracking error in $x_{1}$ i.e. $x_{1 \mathrm{~d}}-x_{1}$ 
$s$

SMC

MFNN

$\boldsymbol{W}, \boldsymbol{V}$

6-DOF

$\operatorname{sgn}(x)$

sat $(x)$ sliding surface

Sliding Mode Control

Multilayer Feedforward Neural Network

weight matrices of output and hidden layers

six degrees-of-freedom

signum function

saturation function

\section{Actuator position and rate saturation limits}

$\delta_{\mathrm{e}}$

$\delta_{\mathrm{a}}, \delta_{\mathrm{r}}$

$\pm 25^{\circ} ; \pm 60 \%$

$\delta_{\mathrm{p}}, \delta_{\mathrm{y}}$

$\pm 25^{\circ} ; \pm 90 \%$

$\pm 25^{\circ} ; \pm 80 \% \mathrm{~s}$

Geometric and mass properties of the aircraft $[7,8]$

Mass

Length

$24000 \mathrm{~kg}$

Wingspan

$17.20 \mathrm{~m}$

Wing surface area

$11.80 \mathrm{~m}$

Mean chord

$39.90 \mathrm{~m}^{2}$

Roll-axis moment of inertia

$4.4 \mathrm{~m}$

Pitch-axis moment of inertia

$3.512 \times 10^{4} \mathrm{~kg} \mathrm{~m}^{2}$

$2.643 \times 10^{5} \mathrm{~kg} \mathrm{~m}^{2}$

Yaw-axis moment of inertia

$2.911 \times 10^{5} \mathrm{~kg} \mathrm{~m}^{2}$

\section{Symmetric 6-DOF Dynamics in Body Axes [20]}

The force and moment balance equations for a standard symmetric aircraft are given by

$$
\begin{gathered}
{\left[\begin{array}{c}
\dot{u} \\
\dot{v} \\
\dot{w}
\end{array}\right]=\left[\begin{array}{c}
-q w+r v \\
-r u+p w \\
-p v+q u
\end{array}\right]+\left[\begin{array}{c}
-g \sin \theta \\
g \cos \theta \sin \varphi \\
g \cos \theta \cos \varphi
\end{array}\right]+\frac{1}{m}\left[\begin{array}{c}
F_{X} \\
F_{Y} \\
F_{Z}
\end{array}\right]} \\
{\left[\begin{array}{ccc}
I_{x x} & 0 & -I_{x z} \\
0 & I_{y y} & 0 \\
-I_{x z} & 0 & I_{z z}
\end{array}\right]\left[\begin{array}{c}
\dot{p} \\
\dot{q} \\
\dot{r}
\end{array}\right]=\left[\begin{array}{c}
\left(I_{y y}-I_{z z}\right) q r+I_{x z} p q \\
\left(I_{z z}-I_{x x}\right) r p+I_{x z}\left(r^{2}-p^{2}\right) \\
\left(I_{x x}-I_{y y}\right) p q-I_{x z} q r
\end{array}\right]+\left[\begin{array}{c}
M_{\mathrm{X}} \\
M_{\mathrm{Y}} \\
M_{\mathrm{Z}}
\end{array}\right]}
\end{gathered}
$$

\section{Asymmetric 6-DOF Dynamics in Body Axes [1, 2, 6-8]}

The force and moment balance equations for an asymmetric aircraft are given by 


$$
\begin{aligned}
& {\left[\begin{array}{cccccc}
1 & 0 & 0 & 0 & z_{c m} & -y_{c m} \\
0 & 1 & 0 & -z_{c m} & 0 & x_{c m} \\
0 & 0 & 1 & y_{c m} & -x_{c m} & 0
\end{array}\right]\left[\begin{array}{c}
\dot{u} \\
\dot{v} \\
\dot{w} \\
\dot{p} \\
\dot{q} \\
\dot{r}
\end{array}\right]=\left[\begin{array}{l}
-q w+r v+\left(q^{2}+r^{2}\right) x_{c m}-p q y_{c m}-r p z_{c m} \\
-r u+p w+\left(r^{2}+p^{2}\right) y_{c m}-p q x_{c m}-q r z_{c m} \\
-p v+q u+\left(p^{2}+q^{2}\right) z_{c m}-r p x_{c m}-q r y_{c m}
\end{array}\right]+} \\
& +\left[\begin{array}{c}
-g \sin \theta \\
g \cos \theta \sin \varphi \\
g \cos \theta \cos \varphi
\end{array}\right]+\frac{1}{m^{\prime}}\left[\begin{array}{c}
F_{X} \\
F_{Y} \\
F_{Z}
\end{array}\right] \\
& {\left[\begin{array}{cccccc}
0 & -m^{\prime} z_{c m} & m^{\prime} y_{c m} & I_{x x}^{\prime} & -I_{x y}^{\prime} & -I_{x z}^{\prime} \\
m^{\prime} z_{c m} & 0 & -m^{\prime} x_{c m} & -I_{x y}^{\prime} & I_{y y}^{\prime} & -I_{y z}^{\prime} \\
-m^{\prime} y_{c m} & m^{\prime} x_{c m} & 0 & -I_{x z}^{\prime} & -I_{y z}^{\prime} & I_{z z}^{\prime}
\end{array}\right]\left[\begin{array}{c}
\dot{u} \\
\dot{v} \\
\dot{w} \\
\dot{p} \\
\dot{q} \\
\dot{r}
\end{array}\right]=} \\
& =\left[\begin{array}{c}
I_{x z}^{\prime} p q+\left(I_{y y}^{\prime}-I_{z z}^{\prime}\right) q r-I_{x y}^{\prime} r p+I_{y z}^{\prime}\left(q^{2}-r^{2}\right) \\
-I_{y z}^{\prime} p q+I_{x y}^{\prime} q r+\left(I_{z z}^{\prime}-I_{x x}^{\prime}\right) r p+I_{x z}^{\prime}\left(r^{2}-p^{2}\right) \\
\left(I_{x x}^{\prime}-I_{y y}^{\prime}\right) p q-I_{x z}^{\prime} q r-I_{y z}^{\prime} r p+I_{x y}^{\prime}\left(p^{2}-q^{2}\right)
\end{array}\right]+ \\
& +\left[\begin{array}{c}
m^{\prime}(q u-p v) y_{c m}+m^{\prime}(r u-p w) z_{c m} \\
m^{\prime}(p v-q u) x_{c m}+m^{\prime}(r v-q w) z_{c m} \\
m^{\prime}(p w-r u) x_{c m}+m^{\prime}(q w-r v) y_{c m}
\end{array}\right]+\left[\begin{array}{c}
-z_{c m} m^{\prime} g \cos \theta \sin \varphi+y_{c m} m^{\prime} g \cos \theta \cos \varphi \\
-z_{c m} m^{\prime} g \sin \theta-x_{c m} m^{\prime} g \cos \theta \cos \varphi \\
y_{c m} m^{\prime} g \sin \theta+x_{c m} m^{\prime} g \cos \theta \sin \varphi
\end{array}\right]+\left[\begin{array}{c}
M_{x} \\
M_{y} \\
M_{z}
\end{array}\right]
\end{aligned}
$$

\section{References}

[1] BACON, B.J. and GREGORY, I.M. General Equations of Motion for a Damaged Asymmetric Aircraft. In Proceedings of the AIAA Atmospheric Flight Mechanics Conference and Exhibit. Hilton Head: AIAA, 2007, p. 6306-6379. DOI 10.2514/6.2007-6306.

[2] NGUYEN, N., KRISHNAKUMAR, K., KANESHIGE, J. and NESPECA, P. Flight Dynamics and Hybrid Adaptive Control of Damaged Aircraft. Journal of Guidance Control Dynamics, 2008, vol. 31, no. 5, p. 171-186. DOI 10.2514/ 1.28142 . 
[3] SHAH, G. Aerodynamic Effects and Modeling of Damage to Transport Aircraft. In Proceedings of the AIAA Atmospheric Flight Mechanics Conference and Exhibit. Honolulu: AIAA, 2008, p. 1-13. DOI 10.2514/6.2008-6203.

[4] OUEllette, J., RAGHAVAN, B., PATIL, M. and KAPANIA, R. Flight Dynamics and Structural Load Distribution for a Damaged Aircraft. In Proceedings of the AIAA Atmospheric Flight Mechanics Conference. Chicago: AIAA, 2009, p. 1-20. DOI 10.2514/6.2009-6153.

[5] MUKHERJEE, B.K. and SINHA, M. Modeling and Bifurcation Analysis of Combat Aircraft Dynamics under Lateral CM Shift. In Proceedings of the AIAA Atmospheric Flight Mechanics Conference. San Diego: AIAA, 2016, p. 1-16. DOI 10.2514/6.2016-1037.

[6] MUKHERJEE, B.K. and SINHA, M. Dynamic Inversion Control for Performing Herbst Maneuver with Lateral Center-of-Gravity Offset. Defence Science Journal, 2017, vol. 67. no.2, p.198-206. DOI 10.14429/dsj.67.10374.

[7] MUKHERJEE, B.K. and SINHA, M. Extreme Aircraft Maneuver under Sudden Lateral CG Movement: Modeling and Control. Aerospace Science and Technology, 2017, vol. 68, p. 11-25. DOI 10.1016/j.ast.2017.04.030.

[8] MUKHERJEE, B.K. and SINHA, M. Nonlinear Dynamics and Control of a Laterally Mass Varying Fighter Aircraft. Proceedings of the Institution of Mechanical Engineers, Part G: Journal of Aerospace Engineering, 2018, vol. 232, no. 16, p. 3118-3134. DOI 10.1177/ 0954410017723360.

[9] MUKHERJEE, B.K. and SINHA, M. Large Angle Maneuvering with an Asymmetric Aircraft: A Single Loop Control Formulation. In Proceedings of the AIAA Guidance, Navigation, and Control Conference. Kissimmee: AIAA, 2018. DOI 10.2514/6.2018-1869.

[10] SNELL, S.A., ENNS, D.F. and GARRARD Jr, W.L. Nonlinear Inversion Flight Control for a Supermaneuverable Aircraft. Journal of Guidance, Control, and Dynamics, 1992, vol. 15, no. 4, 1992, p. 976-984. DOI 10.2514/3.20932.

[11] KHATRI, A.K., SINGH, J. and SINHA, N.K. Accessible Regions for Controlled Aircraft Maneuvering. Journal of Guidance, Control, and Dynamics, 2013, vol. 36, no. 6, p. 1829-1834. DOI 10.2514/1.59592.

[12] BUGAJSKI, D.J. and ENNS, D.F. Nonlinear Control Law with Application to High Angle-of-Attack Flight. Journal of Guidance, Control, and Dynamics, 1992, vol. 15, no. 3, p. 761-767. DOI 10.2514/3.20902.

[13] KIM, B.S. and CALISE, A.J. Nonlinear Flight Control Using Neural Networks. Journal of Guidance, Control, and Dynamics, 1997, vol. 20, no. 1, p. 2633. DOI 10.2514/2.4029.

[14] SUN, T., PEI, H., PAN, Y., ZOU, H. and ZHANG, C. Neural Network-Based Sliding Mode Adaptive Control for Robot Manipulators. Neurocomputing, 2011, vol. 74, no. 11-15, p. 2377-2384. DOI 10.1016/j.neucom.2011.03.015.

[15] CARTER, B.R. Time-Optimization of High Performance Combat Maneuvers [MS Thesis]. Monterey: Naval Postgraduate School, 2005. 244 p. [viewed 201802-15]. Available from https://core.ac.uk/download/pdf/36695907.pdf.

[16] CHIANG, R.Y., SAFONOV, M.G., HAIGES, K., MADDEN, K. and TEKAWY, J. A Fixed $\mathrm{H}^{\infty}$ Controller for a Supermaneuverable Fighter Performing the Herbst 
Maneuver. Automatica, 1993, vol. 29, no.1, p. 111-127. DOI 10.1016/00051098(93)90176-t.

[17] VINAYAGAM, A.K. and SINHA, N.K. An Assessment of Thrust Vector Concepts for Twin-Engine Airplane. Proceedings of the Institution of Mechanical Engineers, Part G: Journal of Aerospace Engineering, 2014, vol. 228, no. 6, p. 960-979. DOI 10.1177/0954410013485697.

[18] SESHAGIRI, S. and PROMTUN, E. Sliding Mode Control of F-16 Longitudinal Dynamics. In Proceedings of the American Control Conference. Seattle: IEEE, 2008, p. 1770-1775. DOI 10.1109/ACC.2008.4586748.

[19] SLOTINE, J.J. and LI, W. Applied Nonlinear Control. New Jersey: PrenticeHall, 1991, 459 p. ISBN 978-0-13-040890-5.

[20] STEVENS, B.L. and LEWIS, F.L. Aircraft Control and Simulation. $2^{\text {nd }}$ Edition, New Jersey: Wiley, 2003, 664 p. ISBN 978-0-471-37145-8.

[21] DEMUTH, H. and BEALE, M. Neural Network Toolbox: For Use with MATLAB. MathWorks Inc., 2002, 840 p. [viewed 2019-01-15]. Available from http://www.image.ece.ntua.gr/courses_static/nn/matlab/nnet.pdf. 